\title{
Ileosigmoid Knotting - A Rare Case Report
}

\author{
M. Sabari Girieasen ${ }^{*}$, Naveenkumar Viswanathan, S. Nedunchezhian, Kannan Ross
}

Institute of General Surgery, Madras Medical College, Chennai, Tamilnadu, India

DOI: $10.36347 /$ sjmcr.2020.v08i02.027

| Received: 13.02.2020 | Accepted: 20.02.2020 | Published: 22.02 .2020

*Corresponding author: Dr. M. Sabari Girieasen

\section{Abstract}

Ileosigmoid knotting is a rare cause of acute intestinal obstruction where the ileum wraps around the base of sigmoid colon and threatens the viability of both ileum and sigmoid colon. We had a case of 65 year old male who presented with symptoms of intestinal obstruction. His vitals were stable with no signs of peritonitis. Imaging showed intestinal obstruction with possibility of volvulus. Emergency laparotomy was done and there was gangrenous ileum wrapped around the gangrenous sigmoid colon. Gangrenous segments were resection and colorectal anastomosis and double barrel ileostomy were done. Our case is categorized as type I ileosigmoid knotting with no evidence of shock. Patient improved gradually and he was discharged on POD 10. As the condition was suspected early, active intervention was made which lead to better outcome in this patient. Ileosigmoid knotting should always be considered as differential diagnosis for acute intestinal obstruction in males.

Keywords: Ileum, sigmoid, knotting, ISK, volvulus, obstruction.

Copyright @ 2020: This is an open-access article distributed under the terms of the Creative Commons Attribution license which permits unrestricted use, distribution, and reproduction in any medium for non-commercial use (NonCommercial, or CC-BY-NC) provided the original author and source are credited.

\section{INTRODUCTION}

Ileosigmoidal knotting (ISK), also known as compound volvulus or double volvulus, is a rare cause of acute intestinal obstruction, which is likely to occur in developing or underdeveloped countries. Its incidence in the general population is not known. ISK is common in adult males, and the peak incidence is in the fourth decade [1]. Mortality varies from 0 to $47 \%$.

Here the ileum wraps around the base of the sigmoid colon and forms a pseudoknot. Strangulation caused by the "knot" threatens the viability of both ileum and sigmoid colon. Emergency surgery is the only therapeutic option available, and it is life-saving. ISK is often a diagnostic surprise to the operating surgeon. Preoperative diagnosis is extremely rare [2].

Here we report a rare case of ileosigmoid knotting in a 65 year male whose early detection and adequate treatment has led to better outcome.

\section{CASE REPORT}

A 65 year old male presented to the emergency department of Madras Medical College with complaints of abdominal pain, abdominal distension and vomiting for one day. Patient developed abdominal pain before one day which was acute in onset, diffuse, continuous and associated with abdominal distension. He also has bilious vomiting several episodes and there is no relief of pain after vomiting. He had similar complaints in the past when the pain relievers by itself. He is a chronic smoker and alcoholic with no comorbidities.

On admission, patient was dehydrated with a pulse rate of 104/min and blood pressure of 110/80 mm hg. On abdominal examination, abdomen distended and diffuse tenderness was present with guarding and no rigidity. Shifting dullness was noted. Per rectal examination was empty and there was no faecal staining.

Routine blood investigations were normal and viral markers were negative. $X$ ray showed multiple air fluid levels and no pneumoperitoneum. USG showed dilated small bowel loops and possibility of intestinal obstruction. CT abdomen showed swirled appearance of ileum with dilated proximal loops and collapsed distal loops and gave the possibility of volvulus. Free fluid was noted.

\section{RESULTS}

Emergency laparotomy was planned. Following findings noted. About one litre of haemorrhagic fluid was drained. Ileal loops were found to encircle the sigmoid colon. Both the ileal loops and sigmoid colon was gangrenous (Figure 1 and 2) and hence proceeded with resection of gangrenous sigmoid colon and ileum. 
About $60 \mathrm{~cm}$ of ileum $5 \mathrm{~cm}$ proximal to the ileocaecal junction was gangrenous, resected and double barrel ileostomy was placed in the right iliac fossa (Figure 4). Then about $15 \mathrm{~cm}$ of gangrenous sigmoid colon was resected and colorectal anastomosis was done (Figure 3). Abdomen closed in layers. Stoma started functioning by POD 2. Drain was removed on POD 5 and started on orals. Patient improved and discharged on POD 10.

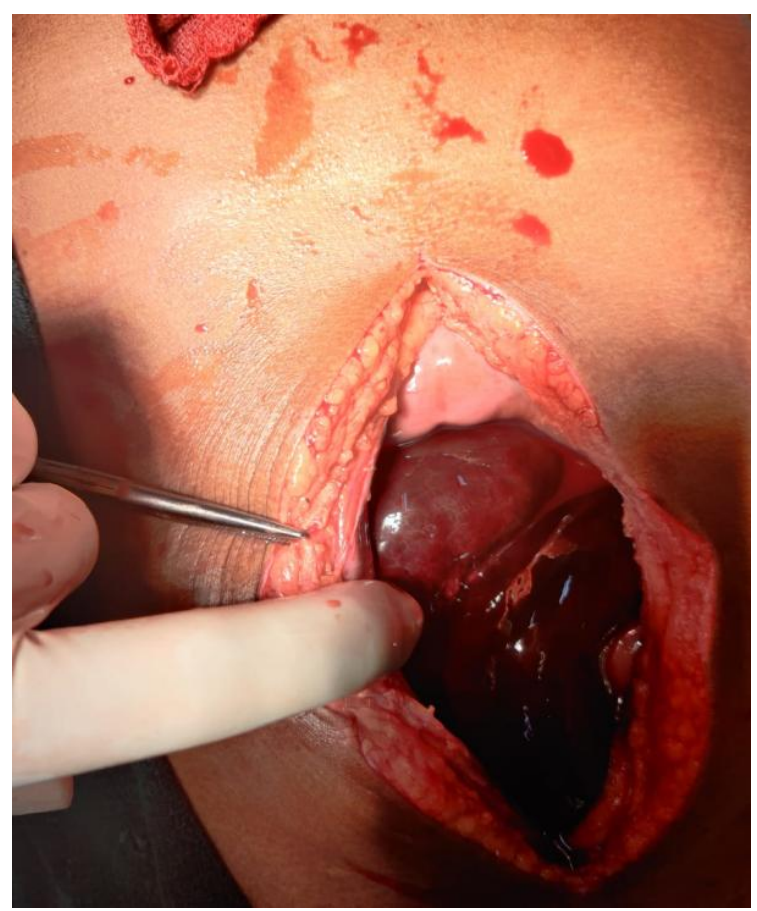

Fig-1: Intra operative picture on opening the peritoneum

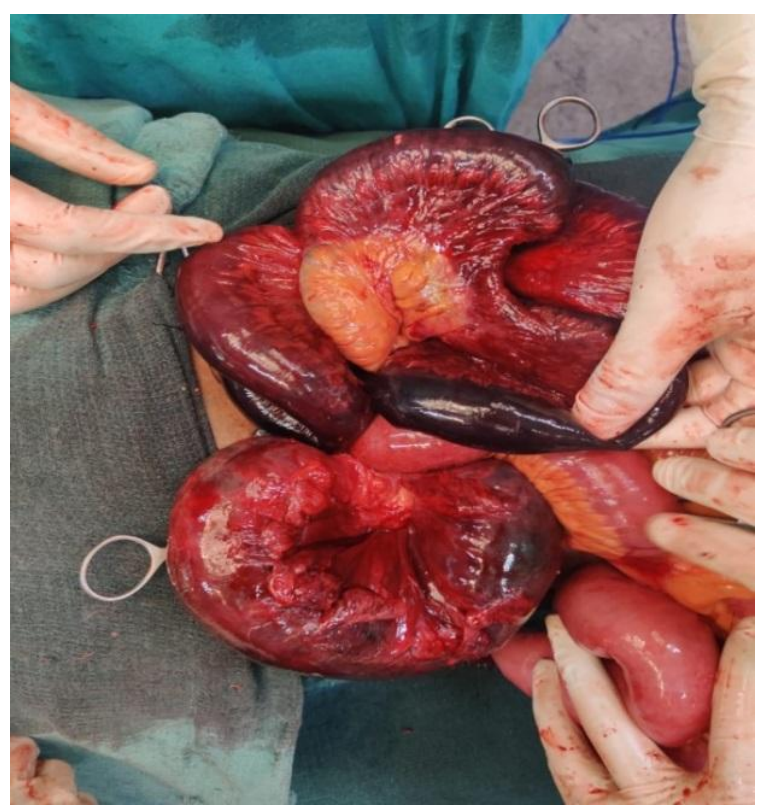

Fig-2: Gangrenous ileal loops above and gangrenous sigmoid colon below

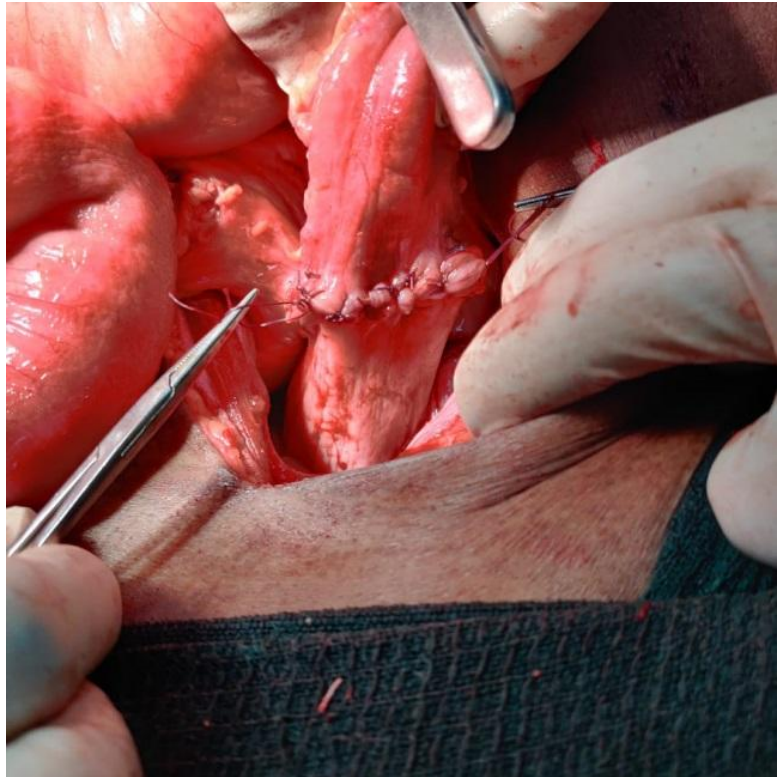

Fig-3: Colorectal anastomosis

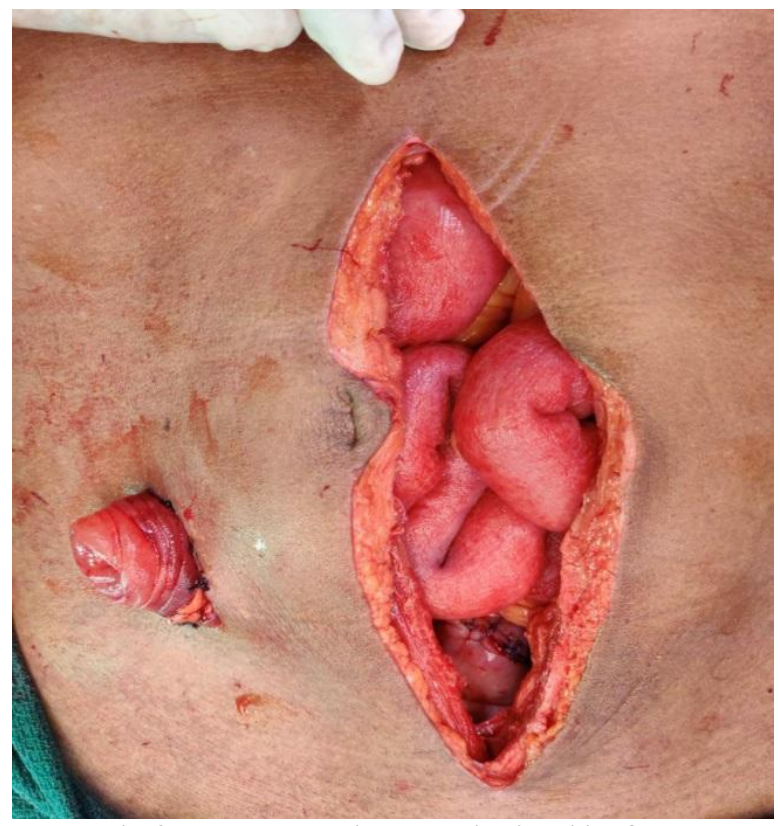

Fig-4: Double barrel ileostomy in right iliac fossa

\section{DiSCUSSION}

Ileosigmoid knotting is a rare cause of acute intestinal obstruction where the ileum wraps around the base of sigmoid colon and threatens the viability of both ileum and sigmoid colon. Predisposing factors described are high fiber diet, frail and debilitated patients, chronic laxative use, hyper mobile small intestine with markedly elongated mesentery. Primary event is usually a hyperactive ileum winding around the narrow pedicle of a passive sigmoid [3].

Alver et al. arbitrarily categorized the types of ISK as follows: Type 1-- The ileum (active component) wraps itself around the sigmoid colon (passive component) in a clockwise or anticlockwise direction. Type II--The sigmoid colon (active component) wraps 
itself around a loop of ileum (passive component) in a clockwise or anticlockwise direction. Type III-- The ileocecal segment (active component) wraps itself around the sigmoid colon (passive component). Our case belongs to type I where the ileum wraps the sigmoid colon [4]. CT shows whirl sign but the preoperative diagnosis is unlikely in ISK.

In the new classification, the patients with ISK are classified as follows: Class 1, patients with no risk factor (advanced age, associated disease); Class 2, those with no shock or bowel gangrene but other risk factors mentioned above; Class3, those with shock; Class 4, those with ileum or sigmoid colon gangrene; Class 5, those with both shock and ileum or sigmoid colon gangrene; Class 6 , those with both ileum and sigmoid colon gangrene [5]. Our case belongs to class 6 where mortality is high.

Kumar et al. [6] reported a similar case of ISK in a 56 year old male and terminal ileostomy was done as there was gangrene of ileocaecal junction. Mahim Koshariya et al. [3] reported a similar case in a 60 year male where primary anastomosis was done with covering ileostomy. Similar treatment modality was followed for our patient as per the literature. Though our patient had symptoms only for a day, patient ended up with bowel gangrene even after early diagnosis and active management. Morbidity in this patient was highly reduced because of early intervention.

\section{CONCLUSION}

Ileosigmoid knotting should always be considered in the differential diagnosis of male patient with acute intestinal obstruction. If the treatment is delayed, it can rapidly lead to bowel gangrene.

\section{DeClarations}

Funding: The authors have not declared a specific grant for this research from funding agency in the public, commercial or not for profit sectors.

Conflict of interest: none declared

Patient consent for publication: Obtained.

\section{REFERENCES}

1. Diallo OR, Camara SA, Bah AT, Barry M, Cisse A. Le noma chez les enfants infectes par le VIH au CHU de Conakry: 5 cas.

2. Raveenthiran V. The ileosigmoid knot: new observations and changing trends. Dis Colon Rectum. 2001;44:1196-2000.

3. Koshariya M, Choudhary A, Gome N, Meravi A, Rai A, Songra MC. Ileosigmoid knotting: An unusual cause of intestinal obstruction.

4. Alver O, Ören D, Tireli M, Kayabaşi B, Akdemir D. Ileosigmoid knotting in Turkey. Diseases of the colon \& rectum. 1993 Dec 1;36(12):1139-47.

5. Atamanalp SS, Oeztuerk G, Aydinli B, Yildirgan MI, Başoğlu M, Oeren D, KANTARCI AM. A new classification for ileosigmoid knotting. Turkish Journal of Medical Sciences. 2009 Sep 23;39(4):541-5.

6. Kumar R, Kenchappa PK, Meena K, Singh BK. Ileosigmoid knotting: an unusual cause of acute intestinal obstruction with bowel gangrene. BMJ Case Reports CP. 2019 May 1;12(5):e226663. 